\title{
An Overview of the NASA FAP Hypersonics Project Airbreathing Propulsion Research
}

\author{
A.H.Auslender ${ }^{1}$ \\ NASA Langley Research Center, Hampton, Va. 23681, USA \\ Kenneth L. Suder ${ }^{2}$ and Scott R. Thomas ${ }^{3}$ \\ NASA Glenn Research Center, Cleveland, OH 44135, USA
}

\begin{abstract}
The propulsion research portfolio of the National Aeronautics and Space Administration Fundamental Aeronautics Program Hypersonics Project encompasses a significant number of technical tasks that are aligned to achieve mastery and intellectual stewardship of the core competencies in the hypersonic-flight regime. An overall coordinated programmatic and technical effort has been structured to advance the state-of-the-art, via both experimental and analytical efforts. A subset of the entire hypersonics propulsion research portfolio is presented in this overview paper. To this end, two programmatic research disciplines are discussed; namely, (1) the Propulsion Discipline, including three associated research elements: the X-51A partnership, the HIFiRE-2 partnership, and the Durable Combustor Rig, and (2) the Turbine-Based Combine Cycle Discipline, including three associated research elements: the Combined Cycle Engine Large Scale Inlet Mode Transition Experiment, the small-scale Inlet Mode Transition Experiment, and the High-Mach Fan Rig.
\end{abstract}

\begin{tabular}{llll}
\multicolumn{5}{c}{ Nomenclature } \\
AFRL & $=$ Air Force Research Laboratory & PAES & $=$ Planetary Atmospheric Entry Systems \\
AHSTF & $=$ Arc-Heated Scramjet Test Facility & RALV & $=$ Reusable Airbreathing Launch Vehicle \\
AFOSR & $=$ Air Force Office of Scientific Research & SWT & $=$ Supersonic Wind Tunnel \\
CCE & $=$ Combined Cylce Engine & TBCC & $=$ Turbine-Based Combined Cycle \\
CFD & $=$ Computational Fluid Dynamics & TDLAS & $=$ Tunable Diode Laser Absorption System \\
DCR & $=$ Durable Combustor Rig & TSTO & $=$ Two-Stage-to-Orbit \\
DSTO & $=$ Australian Defense Science and & $8^{\prime}$ HTT & $=$ \\
& &
\end{tabular}

\section{Introduction}

$\mathrm{T}$ HE "National Aeronautics Research and Development Policy" document, issued by the National Science and Technology Council in December 2006, stated that one, among several, of the guiding objectives of the United States federal aeronautic research and development endeavors shall be stable and long-term foundational research efforts. Nearly concurrently, the National Academies issued a more technically focused aeronautics blueprint, entitled: the "Decadal Survey of Civil Aeronautics - Foundations for the Future." Taken together these documents outline the principles of a baseline aeronautics maturation plan. Thus, in response to these overarching inputs and

\footnotetext{
${ }^{1}$ Associate Principle Investigator of the FAP Propulsion Discipline, AIAA Member

${ }^{2}$ Senior Aerospace Technologist, AIAA Member

${ }^{3}$ Associate Principle Investigator of the FAP Turbine-Based Combined Cycle Discipline
}

1

American Institute of Aeronautics and Astronautics 
others, the National Aeronautics and Space Administration (NASA) via the Fundamental Aeronautics Program (FAP), a program within the NASA Aeronautics Research Mission Directorate, initiated foundational research and technology development research projects to enable the capability of future vehicles to operate across a broad range of Mach numbers, inclusive of the subsonic, supersonic, and hypersonic flight regimes.

Specifically, the FAP Hypersonics Project, addressing the hypersonic flight regime, concentrates on two distinct missions: (1) the Reusable Airbreathing Launch Vehicle (RALV) mission, and (2) the Planetary Atmospheric Entry Systems (PAES) mission. Primarily, the RALV mission focuses on Two-Stage-To-Orbit (TSTO) systems utilizing airbreathing combined-cycle-engine propulsion; whereas, the PAES mission primarily focuses on entry-decent-andlanding technologies, associated with delivering large payloads to (and from) Mars. Two distinct propulsion research entities exist within the FAP Hypersonics Project structure to address propulsion technologies for the RALV mission: (1) the Propulsion Discipline, and (2) the Turbine-Based Combined Cycle (TBCC) Discipline. Collectively, the research activities of both disciplines are aligned to achieve mastery and intellectual stewardship of the core competencies in the hypersonic-flight regime, which ultimately will be required to implement practical systems with highly integrated airframe and propulsion systems. Additionally, to gain access to academic, industrial and other non-NASA technical expertise, both disciplines fund NASA Research Announcement (NRA) grants, and the Propulsion Discipline is additionally supported by a jointly funded AFOSR-NASA National Hypersonics Science Center. Also, although less commonly utilized, external partnerships agreements are employed with both governmental agencies and industrial entities to leverage resource allocations in research areas of mutual technical interest.

The Propulsion Discipline research portfolio is comprised of both analytic and experimental tasks, and is structured into three overarching research topic areas: (1) combustion fundamentals - addressing both hydrogen and hydrocarbon fuels, and test-media effects, (2) flowpath technologies - addressing both planar and three-dimensional configuration (non-planar) technologies, and (3) advanced computational modeling. Likewise, the Turbine-Based Combined Cycle Discipline research portfolio is comprised of analytic and experimental tasks, and is similarly structured into two broad research topic areas: (1) integrated turbine-based, combined-cycle-engine technologies, and (2) integrated design and performance analysis capability development. The resources allocated to these research disciplines are significant, with slightly in excess of fifty NASA full-time staff assigned. Also, additional support is provided by approximately fifteen NRA tasks and a newly formed National Hypersonics Research Center, entitled: "National Center for Hypersonic Combined Cycle Propulsion." Thus, due to the large number of the existing technical tasks, the subsequent text details a limited, but representative group of Propulsion Discipline and Turbine-Based Combined Cycle (TBCC) Discipline activities addressing the propulsion technologies supporting the RALV mission, namely: (1) the X-51A Propulsion Discipline task, (2) the HIFiRE Flight-2 Payload development Propulsion Discipline task, (3) the Durable Combustor Rig (DCR) Propulsion Discipline task, and (4) the Combined-Cycle-Engine (CCE) Large Scale Inlet Mode Transition Experiment (LIMX) TBCC Discipline task, (5) the Inlet Mode Transition Experiment (IMX) TBCC task, and (6) the High Mach Fan Rig TBCC task.

\section{Propulsion Discipline Tasks}

Three significant Propulsion Discipline technical elements are subsequently discussed. Note that the first two tasks are external partnerships between the Air Force Research Laboratory and the NASA-FAP Hypersonics Project, and are complimentary to one another, with each culminating in hydrocarbon-fueled scramjet flight tests; yet, each operating in a distinct speed regime. The third propulsion task presented in this section is a fundamental combustion experiment and is designed to investigate ramjet-to-scramjet mode-transition mechanisms; as well as, being designed both to acquire composite-heat-exchanger data, and to provide a platform for propulsion flow-field diagnostic techniques development.

\section{A. Propulsion Discipline - X-51A}

The X-51A program, administered by the Air Force Research Laboratory (AFRL), is a propulsion-vehicle technology program that will culminate in a series of flight tests. To supplement this hypersonic-propulsion program, via an externalpartnership agreement, the NASA-FAP Hypersonics Project conducted a series of ground tests using the Langley Research Center 8-Foot High Temperture Tunnel Facility ( $\left.8^{\prime} \mathrm{HHT}\right)$. These tests concluded in September 2008. Four broad test objectives were met: (1) verification of the engine start-up procedure and related

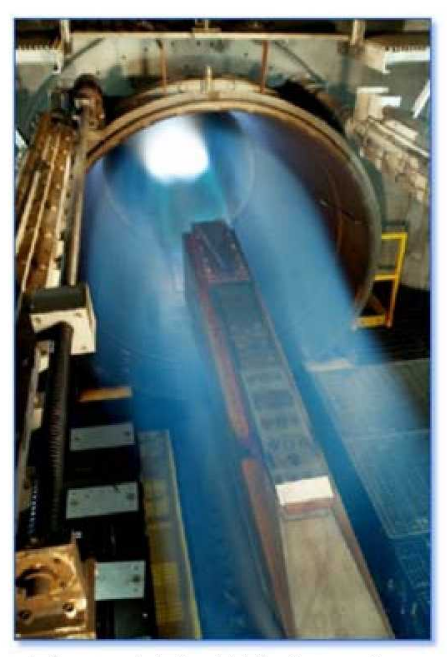

Figure 1. The X51-A hardware (SJX61-2) installed in the NASA LaRC 8'HTT Facility. 
performance sensitivities, with particular emphasis on the thermal management, (2) verification of the performance and operability limits and margins, (3) verification of the control algorithms and associated sensor utilization schemes, and (4) verification of the durability of the engine structure and related components. Additionally, the propulsion-vehicle performance database is being utilized, as part of a Propulsion Discipline research task, in collaboration with AFRL, to assess the predictive performance capability of state-of-the-art computational algorithms, and to investigate test-media-vitiate influences on performance.

\section{B. Propulsion Discipline - HIFiRE Flight-2 Payload}

The HIFiRE program is a collaborative effort between the Australian Defense Science and Technology Office (DSTO) and the United States Air Force Research Laboratory (AFRL). The overarching technical objective is to explore fundamental hypersonic flow physics phenomena through focused flight experiments, which will be used to validate ground test techniques and verify numerical prediction tools. Furthermore, via an additional collaborative agreement between the Air Force Research Laboratory and NASA, the NASA-FAP

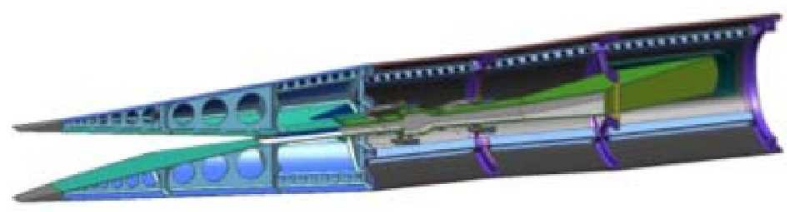

Figure 2. A cross-section of the HIFiRE Flight-2 configuration (inlet and combustor components).

Hypersonics Project has agreed to develop the HIFiRE Flight-2 payload. The primary purpose of this investigation is to extend the hydrocarbon-fueled, flight-derived propulsion database to Mach- 8 total enthalpy conditions; thus, extending the X-51A flight-derived database beyond the anticipated Mach-6+ total enthalpy flight conditions. Also, the acquisition of mode-transition data (from ramjet to scramjet operation) is being sought, as is the entire continuum of engine performance data throughout the Mach-6 to Mach-8 total-enthalpy flight regime. The HIFiRE Flight-2 flowpath is a fixed geometry configuration and has an internal contraction ratio modestly in excess of unity; thereby, ensuring inlet starting (post shroud separation). Furthermore, the propulsion experiment is designed to process a nominal stoichiometric mixture of captured air and injected surrogate fuel, with the latter being a gaseoushydrocarbon blend representative of a thermally stressed JP-7 fuel. Additionally, an in-flight Tunable-Diode-LaserAbsorption-System (TDLAS) will be employed to quantify the mass-flow rate of the captured air, and will be tested during the corresponding NASA-LaRC Arc-Heated-Scramjet-Test Facility (AHSTF) ground-test campaign. Lastly, similar to X-51A tests, the HIFiRE tests will also provide ground-to-flight comparisons allowing for direct assessment of test-media-vitiate influences.

\section{Propulsion Discipline - Durable Combustor Rig}

The Durable Combustor Rig hardware, fabricated for testing in the Direct Connect Supersonic Combustion Test Facility (DCSCTF), addresses two specific propulsion technical objectives, namely: (1) to acquire ramjet-toscramjet mode-transition data for computational and engineering-level algorithm validation and performance assessments, and (2) to provide a platform to enhance the design capability for flight-weight, fuel-cooled, composite-heat-exchanger panels, with the ultimate goal of assessing structural and mechanical design capabilities.

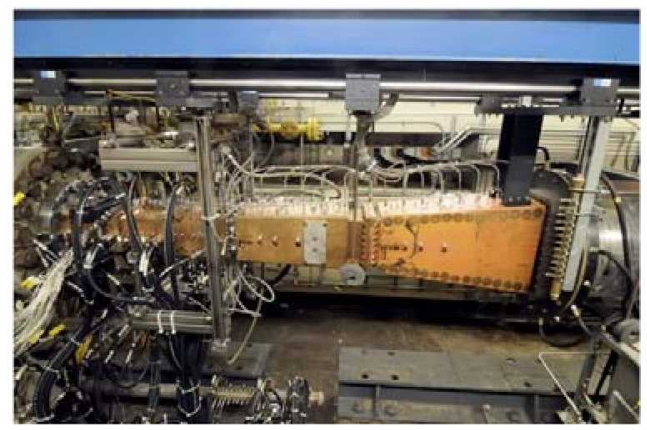

Figure 3(a). The DCR hardware installed in the NASA-LaRC DCSCTF Facility.

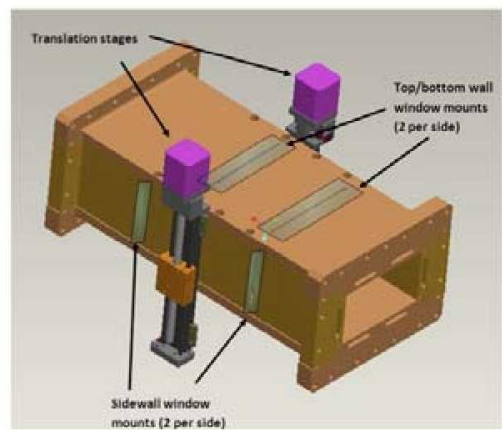

Figure 3(b). A schematic of the TDLAS measurement system (water-tuned version). 
To this end, robust heat-sink hardware has been designed to initially allow for combustor environment characterization and mode-transition research; whereas, actively fuel-cooled hardware has been designed to subsequently acquire composite-heat-exchanger design data. Additionally, in the future, this hardware will be used as a platform for investigating: dynamic seal architectures, high-frequency acoustic loads in relevant propulsion environments, and novel diagnostic techniques (for example, a water-tuned TDLAS measurement).

\section{Turbine-Based Combined Cycle Tasks}

Two significant Turbine-Based Combined Cycle Discipline technical elements are subsequently discussed. These two tasks focus on key combined-cycle engine technical problems, inherent in the integration of the inlet, turbine and additional components; namely, (1) performance quantification, (2) operability margin specification, (3) durability characterization, and (4) structural and thermal constraint identification. Although, note that given the integrated nature of the over-arching problem of enabling the RALV mission, it is not trivial to decouple these technical issues.

\section{A. Turbine-Based Combined Cycle Tasks - Combine Cycle Engine (CCE) Large Scale Inlet Mode Transition Experiment (LIMX)}

A primary focus of the Turbine-Based Combined Cycle Discipline is to develop and validate high-fidelity multidisciplinary and engineering-level tools, which will enable accurate assessments of propulsion system performance, operability, durability, and weight. This technical effort requires a significant experimental and analytic research endeavor, and encompasses the assessment of the state-of-the-art of performance prediction and design codes, inclusive of fluid and turbine performance codes and engineering-level algorithms. Hence, the most significant TBCC Discipline task is the design and test of a large-scale, turbine-based, combine-cycle model. The CCE hardware is in the process of final assembly, and testing is planned to commence early in the 2010 calendar year, at the NASA Glenn Research Center 10'x10' Supersonic Wind Tunnel (SWT). A schematic and a photograph of the partially fabricated model, are presented in Figure 4(a) and Figure 4(b), respectively. Note the test article is approximately thirty feet in length and includes both a forebody plate and a mass-flow-plug assembly, and also, due to the large scale of the test article, the instrumentation density is quite large, and corresponds to a small spatialresolution dimension.

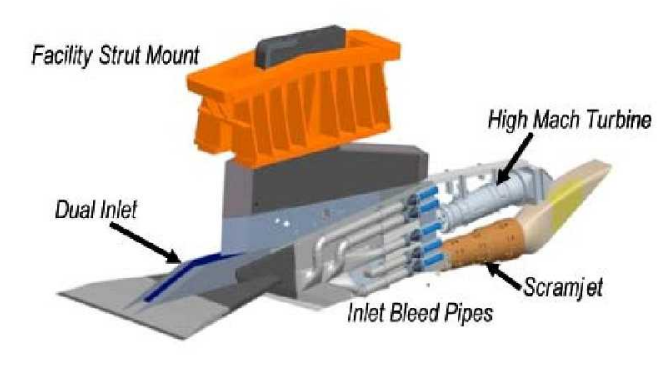

Figure 4(a). A schematic of the CCE hardware.

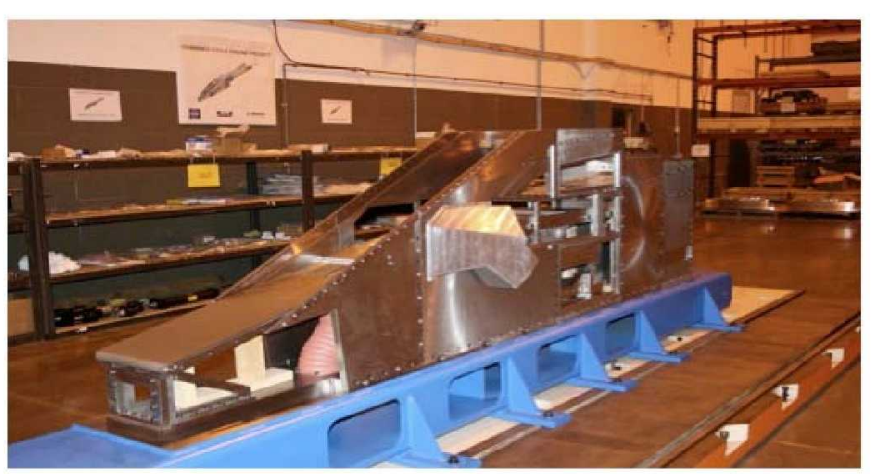

Figure 4(b). The CCE hardware (LIMX components).

The focus of this experimental test effort is to characterize the mode-transition process and to obtain the associated performance constraints, including those applicable to the control algorithms and the associated hardware. Ultimately, this research activity seeks to quantify key combined-cycle engine parameters (specific to the RALV mission); namely, (1) the operating margins of an integrated high-temperature turbine and a dual-mode scramjet engine, (2) integrated dual-inlet operability and performance - inclusive of inlet unstart and operability constraints, distortion constraints, bleed requirements, and control requirements (both software and hardware), (3) mode-transition cycle constraints dictated by the turbine and the ramjet-scramjet flow paths (and associated variable geometry requirements), (4) turbine engine transients (and associated time scales), and lastly, (5) high-altitude turbine engine re-light constraints. Over an approximately three-year time duration there are four distinct, research phases planned; specifically (1) inlet performance and operability characterization, utilizing mechanical throttling (to simulate the combustion process), (2) identification of transient responses for control algorithm development, (3) 
demonstration of mode-transition and inlet unstart-recovery strategies, and (4) complete combined-cycle-engine testing, inclusive of the turbine and aft-end nozzles. Finally, note that this variable-geometry CCE LIMX hardware has been designed for compatibility with a nominally twelve-inch diameter turbine engine.

\section{B. Turbine-Based Combined Cycle Tasks - Small Scale Inlet Mode Transition Experiment (IMX)}

To aid in the definition, characterization, and refinement of a viable TBCC configuration, a series of subscale inlet screening tests were conducted to experimentally explore the baseline TBCC design, and associated operability margins. This was accomplished during a series of tests conducted in the NASA Glenn Research Center (GRC) 1'x1' Supersonic Wind Tunnel (SWT) facility utilizing an inlet model, denoted as the small-scale Inlet Mode Inlet Mode Transition Experiment (IMX). Also, there was extensive computational fluid dynamic (CFD) analysis performed to support this experiment. These studies included CFD simulations of both the low-speed and the highspeed configurations, consistent with the IMX hardware and the relevant GRC-1'x1' test facility conditions; as well as, CFD studies of the LIMX hardware at the relevant GRC 10'x10' Supersonic Wind Tunnel Facility test conditions. Subsequently displayed are both the Mach-4 configuration (with both flow paths open) and the Mach-7 configuration (with only the high speed flow path open), achieved by implementation of the variable-geometry features of this inlet design. Also, a CFD-derived, Mach-4 flow field is presented to indicate the degree of boundary layer migration inherent in TBCC inlet systems, consistent with a highly back-pressured combustor environment.

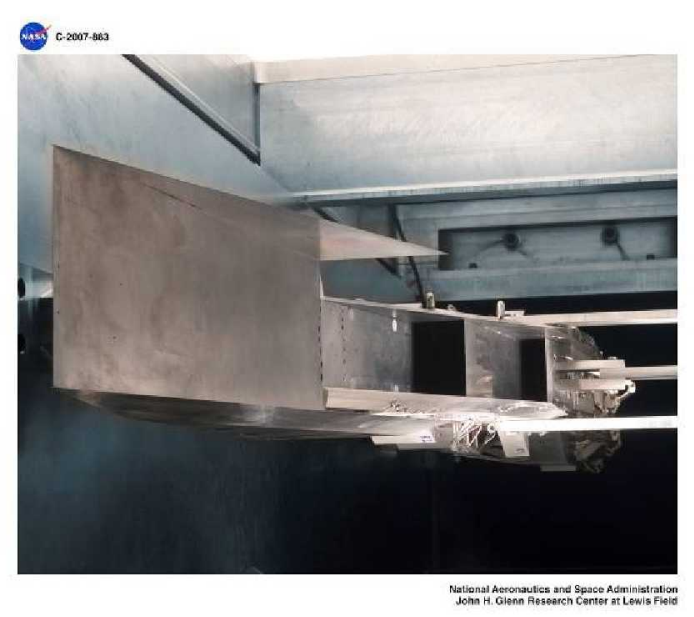

Figure 5.(a) The IMX model Mach-4 configuration installed in the NASA-GRC 1'x1' SWT Facility.

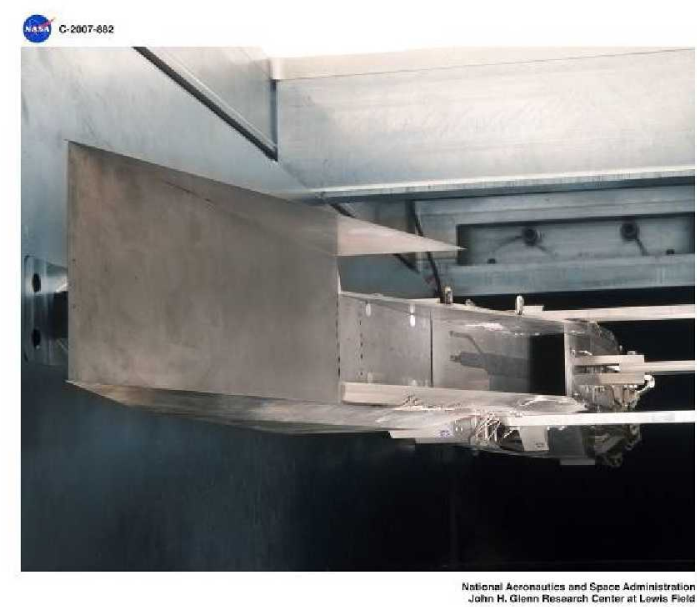

Figure 5. (b) The IMX model Mach-7 configuration installed in the NASA-GRC 1'x1' SWT Facility.

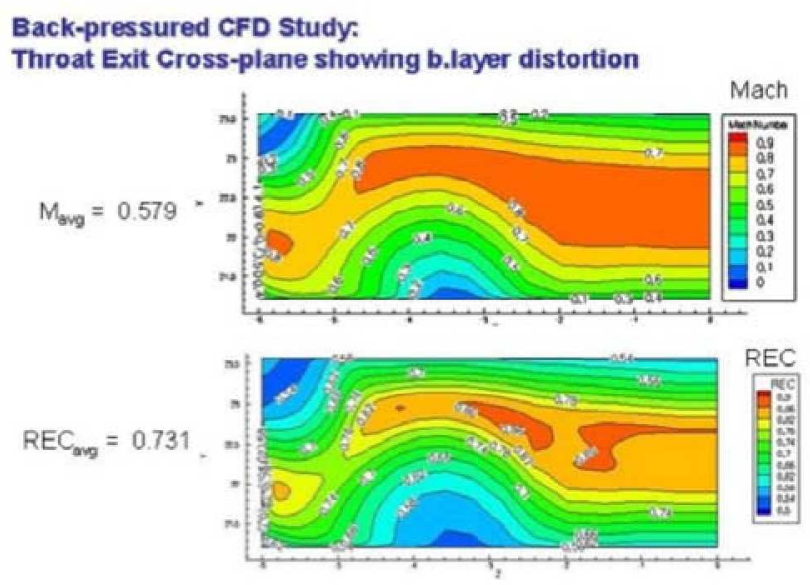

Figure 6. Mach-4 Inlet Recovery and Mach Number Contours 


\section{Turbine-Based Combined Cycle Tasks - High Mach Turbine Engine Fan and Compressor}

Compared to conventional turbine engines, the TBCC-turbine engine will be required both to operate at higher inlet temperatures and operate over a significantly wider speed range. The fan stage, a key component of the TBCCturbine engine, must accommodate these large variations, inclusive of inlet distortions (Figure 6.). To this end, the NASA-FAP Hypersonics Project, and in particular the TBCC Discipline, has established a series of research tasks to characterize TBCC-turbine engine, fan-stage aerodynamic and aeromechanic performance and stability margins, over a wide operating range including both power-on and "windmill" conditions. Additionally, the TBCC Discipline will perform an assessment of the state-of-the-art in fan-stage design, and associated analysis tools applicable to high-speed TBCC propulsion system operation. To meet these objectives, the planned research tasks are: (1) perform aerodynamic and aeromechanical scaling of a relevant state-of-the-art, Mach-4, turbine-engine fan stage and incorporate facility-interface hardware for sub-scale testing in NASA high-speed compressor test facilities, (2) perform pre-test performance and operability analyses, (3) develop relevant fan stage performance "maps" and measure the stall-line stability boundary (over wide range of engine operating conditions), (4) quantify the relevant fan aeromechanics by identifying vibration and flutter boundaries, and assess the ability of state-of-the-art tools to predict flutter mechanisms, (5) quantify inlet-engine interactions by incorporating inlet distortions, and lastly, (6) utilize the existing test articles to generate data to improve the capability of analytic models to assess both off-design performance and operability margins. To date the TBCC discipline has partially accomplished the desired outcomes; namely, (1) a fan stage from a state-of-the-art, Mach-4 turbine engine ${ }^{3}$ has been scaled and fabricated for testing in a NASA fan-test facility, (2) computational fluid dynamic, pre-test predictions have been completed for the uniform inlet flow for the Mach-4 fan stage design, (3) experimental data have been acquired for the fan stage, corresponding to flight conditions at take-off, transonic, Mach-2, Mach-3, Mach-3.3 and Mach-4 conditions, and lastly, (4) computational fluid dynamic, pre-test predictions have been compared with experimental results at design operating points along the flight trajectory and have been found to compare well; whereas, comparisons with off-design data indicate discrepancies and will require further investigation. Furthermore, note that the TBCC Discipline is planning future research tasks to quantifying the sensitivity of fan-stage performance to inlet flow field distortions. This task is coordinated with the CCE LIMX test series, also designed to obtain inlet distortion data. Additionally, these distortion patterns (and levels) will be simulated using distortion screens during future fan-stage testing; thus, allowing for an evaluation of fan-stage performance and operability utilizing realistic conditions.
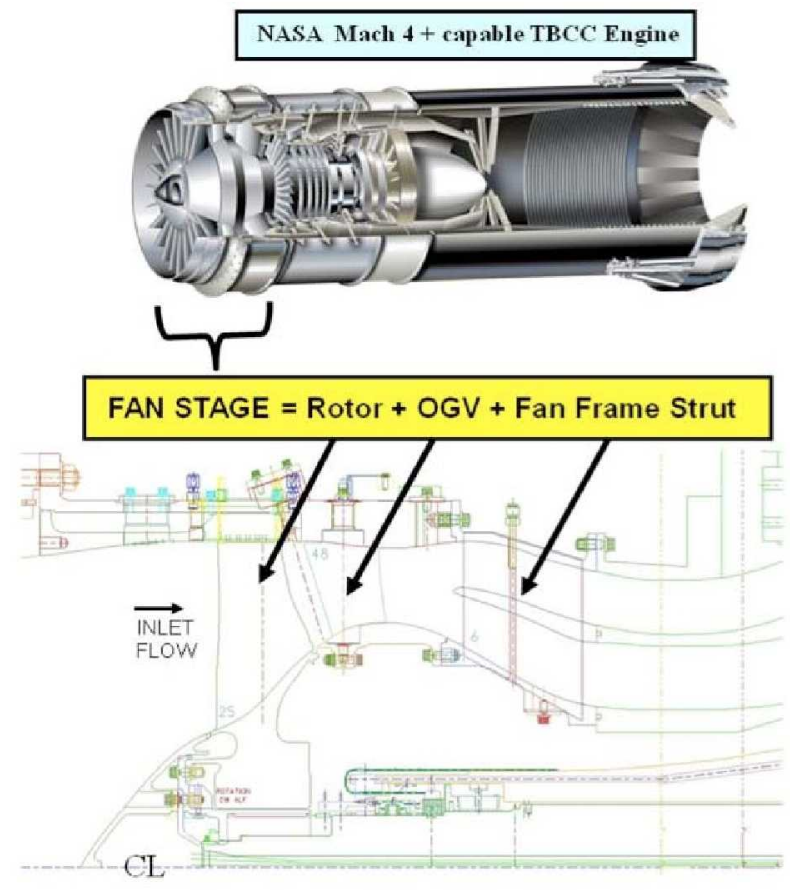

Figure 7. A Fan-Stage flowpath derived from a Mach-4 turbine engine. 


\section{Conclusion}

The Propulsion and Turbine-Based Combined Cycle Disciplines encompass a significant number of technical tasks aligned to achieve mastery and intellectual stewardship of the core competencies in the hypersonic flight regime. Together the disciplines form a coordinated effort, to acquire experimental data and perform analyses on relevant propulsion configurations and unit problems. Ultimately, this has been done, in order to both advance, and quantify the shortcomings, of the state-of-the-art in propulsion performance assessment and propulsion design capability, relevant to the NASA FAP Hypersonics Reusable Airbreathing Launch Vehicle mission.

\section{Acknowledgments}

The authors wish acknowledge the Principal Investigator and Lead Scientist, Dr. James L. Pittman and Dr. F. Mcneil Cheatwood, respectively, under whose supervision the NASA FAP Hypersonics Project research activities are conducted.

\section{References}

${ }^{1}$ Marburger, John H., et al., "National Aeronautics Research and Development Policy," National Research and Technology Council, December 2006

2 “'Decadal Survey of Civil Aeronautics - Foundations for the Future," National Academies, June 2006

${ }^{3}$ Shafer D.G., McNelis N., "Development of a Ground Based Mach 4+ Revolutionary Turbine Accelerator Technology Demonstrator (RTATD) for Access to Space" ISOABE 2003 